\title{
Reduction of ocular muscle torque by splitting of the rectus muscle II: Technique and results
}

\author{
R Hoerantner, S Priglinger, T Haslwanter
}

Br J Ophthalmol 2004;88:1409-1413. doi: 10.1136/bjo.2004.042721

Aim: To present the results of a new technique that the authors have developed to weaken the extraocular muscles. The biomechanics of this technique, which is termed "Y-split recession," are given in the companion paper.

Methods: A retrospective study, testing the effects of a new surgical technique on strabismus, nystagmus, and visual acuity. 228 patients (aged 6.8 (SD 6.0) years) with variable angle strabismus and nystagmus were treated by splitting and detaching two rectus muscles, and re-attaching the two halves at an angle of about $65^{\circ}$ to each other. Subjects were examined with the usual orthoptic tests immediately after the operation, and up to 96 months later.

Results: The operation reduced the strabismus, eliminated or weakened the nystagmus in primary position, and improved binocular vision and the development of visual acuity. Only minor side effects were observed.

Conclusions: The Y-split recession can be used to reduce the torque for extraocular rectus muscles, and provides an alternative to "Cüppers Fadenoperation," recession, and similar procedures.

l n most cases, strabismus in children can be corrected by a simple bilateral ocular muscle surgery. However, there are two types of childhood strabismus where this approach does not work: "infantile esotropia," and the rarely occurring "constant congenital esotropia" (synonymous with "nystagmus blocking syndrome"). Both are characterised by a variable strabismus angle, and are commonly treated in the same way.

In our study we have been able to include 211 patients with infantile esotropia and 17 cases of nystagmus blocking syndrome. Preoperatively, all patients used spectacles or bifocal glasses to stabilise accommodative esotropia, and visual acuity had been improved as far as possible for both eyes. However, because of the characteristics of infantile esotropia and constant congenital esotropia, this treatment with fully corrected or bifocal glasses or stabilisation with prisms was insufficient, and surgical intervention was necessary.

Different types of treatment can be used for variable angle strabismus. One is a recession of one or two affected muscles. As described in the companion paper ${ }^{1}$ this change leads to a relaxation of the muscle, thereby reducing the esotropia. However, the symmetrical weakening of both medial rectus muscles also creates an incomitance in sideward gaze, a reduction of adduction. This surgical approach is often used. ${ }^{23}$ A muscle recession of less than $7 \mathrm{~mm}$ avoids incomitance and reduces the minimum strabismus angle, but the variability of the strabismus angle and the nystagmus remain unchanged.

A second well known technique for the treatment of variable angle strabismus is the so called "Fadenoperation" by Cüppers, also referred to as "posterior fixation" or "retroequatorial myopexy." ${ }^{14-7}$ While this technique can also be used for other kinds of strabismus, here we only compare it with respect to its indication for variable angle strabismus. The Cüppers technique leads to a reduction of muscle torque, and has been used clinically for about 30 years. While in most cases the treatment of the variable angle strabismus with this technique is successful, a number of negative side effects have been reported: incomitance in eye movement, retinal detachment, perforation of the sclera during the operation. Bad results have also been reported in myopic or aphakic patients, and in subjects with only one good seeing eye. ${ }^{8-12}$ With the Fadenoperation, the global and orbital layers of the rectus muscles are typically kept together, and both the muscle and the surrounding muscle sheets are sutured to the globe. As a result the incomitance increases. ${ }^{13}$ To avoid the drawbacks of the Fadenoperation, we have tried the new surgical technique presented here.

\section{METHODS}

Before the operation we recorded factors like globe length and corneal diameter. The remaining factors (for example, the distance of the muscle insertion to the limbus) were determined during surgery.

\section{Surgical procedure}

Firstly, the muscle is split bluntly along a length of $15 \mathrm{~mm}$, as shown in figure 1A. To obtain the correct new insertion points for the two muscle halves, we employed the procedure indicated in the side view in figure 2 (for a better overview, see also fig 5 in the companion paper ${ }^{1}$ ): a first orientation point, labelled " $\mathrm{A}$ " in figure $2 \mathrm{~A}$, is located in the middle of the natural insertion of the muscle. A second point, labelled "B," is $6 \mathrm{~mm}$ straight behind A. With the pair of compasses centred at $\mathrm{A}$, the distance " $r_{A}$ " is marked on the globe with methylene blue, as shown in figure $2 \mathrm{~A}$. The same procedure is repeated from $\mathrm{B}$, with the distance " $r_{B}$." The intersection point of the two methylene blue lines marks the new insertion point of the first muscle half. To obtain the insertion point for the second muscle half the same procedure is performed on the other side, as illustrated in figure $2 \mathrm{~A}$.

To ensure that the marks are placed correctly, we suggest measuring the minimum distance between the cornea limbus and the new insertion ("control distance," fig 2B). It provides a simple check if the two new insertions of the muscle parts are correct. The Y-split recession operation is usually performed on both rectus muscles.

This technique simultaneously includes a torque reduction as well as a muscle recession. Note that a split of $15 \mathrm{~mm}$, as performed during surgery (fig 1, top row) does not affect the pulley structure, since the pulleys are located posterior the muscle split. ${ }^{14}$ This is clearly visible in the postmortem dissection (fig l, bottom row), where the muscle has been split $15 \mathrm{~mm}$ to reach the pulley. In the current case, the 

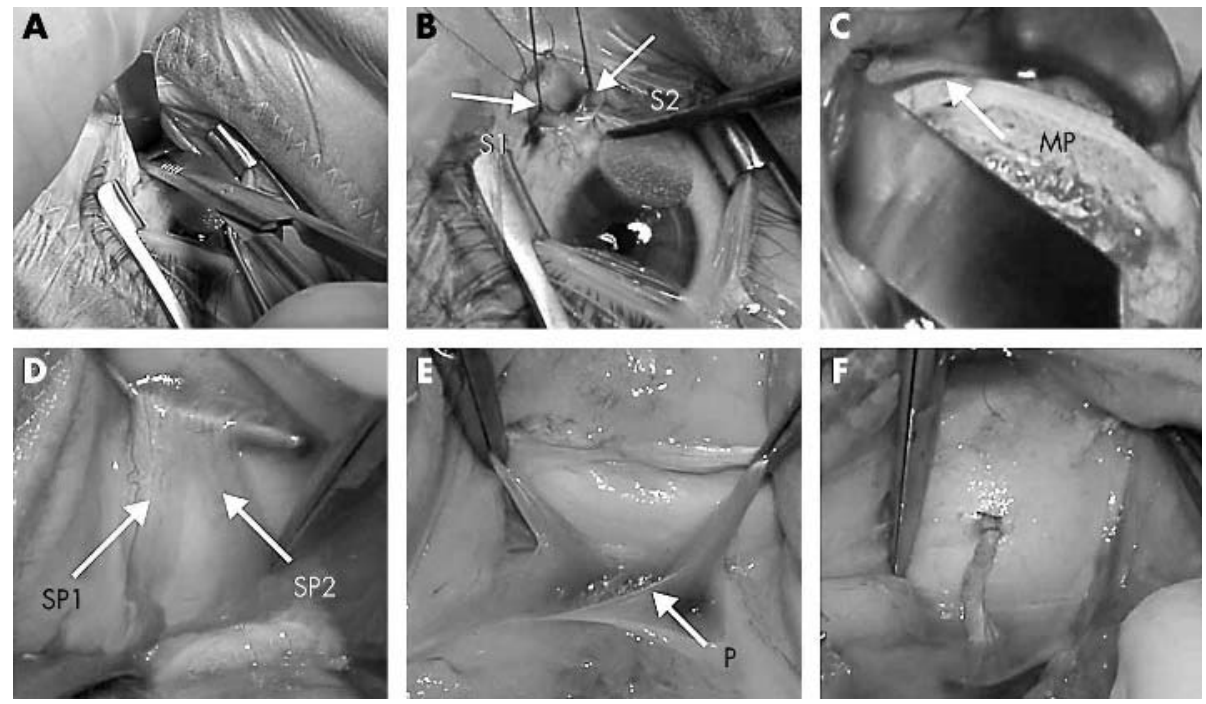

Figure 1 Top row-surgical technique. These pictures were taken during an operation. (A) Firstly, the rectus muscle is split with scissors. (B) Both muscle parts are then fixed by two sutures, S1 and S2. (C) Muscle parts (MP) are re-attached to the globe. Bottom row-postmortem anatomy, of a different subject. (D) Split rectus muscle (SP1 and SP2). (E) Here the muscle is split-for demonstration purposes-along a length of $15 \mathrm{~mm}$. Even with such a long split, the pulley (P) would not be affected. (F) Reinserted muscle part.

pulley location, which varies between subjects, was $15 \mathrm{~mm}$ behind the insertion point of the muscle.

\section{Surgical parameters}

The biomechanical ideas underlying this model are presented in the companion paper. ${ }^{1}$ Based on our muscle model, we chose factors that correspond to a torque reduction of about $26 \%$. This torque reduction is comparable with the torque reduction by a Fadenoperation, using a suture $15 \mathrm{~mm}$ behind the insertion. Depending on the amount of torque reduction, the Y-split recession technique can be used to achieve a number of effects, from avoiding a palsy to creating one. In the present case, it was chosen such that it prevents incomitance through a fairly constant torque reduction.

Note that a Y-split recession inherently combines two effects: by reducing the lever arm, we reduce the torque exerted by the muscle for all eye positions, thereby reducing the variability of the strabismus angle. The muscle recession controls the minimum strabismus angle: by changing the

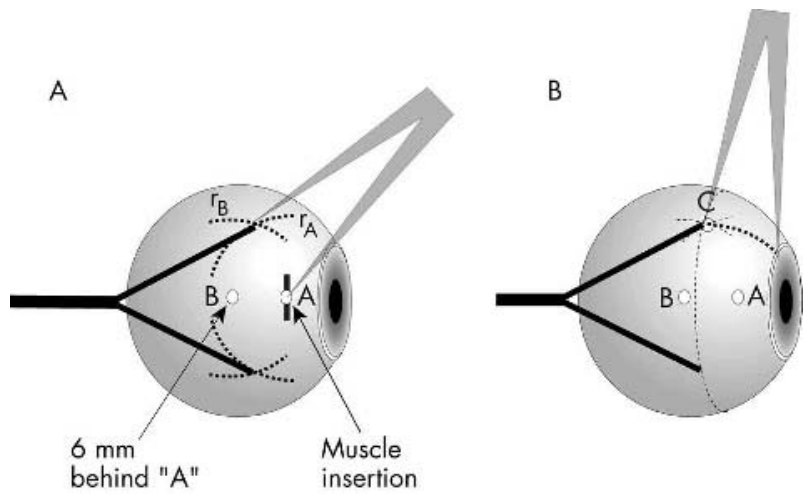

Figure 2 Y-split recession, side view. (A) The first orientation point $\left(" \mathrm{~A}^{\prime \prime}\right)$ is given by the middle of the natural muscle insertion. The second orientation point $\left(" \mathrm{~B}^{\prime \prime}\right)$ is located $6 \mathrm{~mm}$ straight behind $\mathrm{A}$. With a compass, the distance $r_{A}$ is marked with colour on the globe. The same procedure is repeated from $B$, with the distance $r_{B}$. The intersection of the two marked lines indicates the new insertion points for the split muscle halves. (B) The "control distance" ensures correct placement of the new insertion points. muscle recession, the primary position of the eye can be controlled. On average, the following values were used for a globe length of $21.2 \mathrm{~mm}$ :

$$
\mathrm{r}_{\mathrm{B}}=8.4-0.02 * \text { StrabAngle }(\mathrm{mm})
$$

where StrabAngle is the maximum strabismus angle. The range of $r_{A}$ was $6.9-13.7 \mathrm{~mm}$, and was $6.6-11.8 \mathrm{~mm}$ for $r_{B}$.

The resulting muscle recession is given by

Recession $=-2.0-0.052$ * StrabAngle $(\mathrm{mm})$,

and the control distance by

ControlDistance $=10.2+0.032{ }^{*}$ StrabAngle $(\mathrm{mm})$.

Subjects with a minimal strabismus angle above $23^{\circ}$ need an additional recession or plication of the lateral rectus muscle to fully compensate for the complete strabismus angle.

\section{Subjects and test parameters}

Our study includes 228 patients that have been operated with the Y-split recession technique. Before the operation, the patients or their legal representatives were informed about possible side effects, and written consent for the operation was obtained. We also tried to optimise amblyopic therapy before surgery. As a result, the average age was rather high, mean 6.8 (SD 6.0) years (range 1.3-46.8 years).

Subjects with albinism, severe nystagmus, and some other neurological diseases did not reach full visual acuity. Patients with remaining amblyopia were not treated with Y-split recession until they had reached their maximum visual acuity. All patients were treated with bilateral medial rectus surgery.

Tests were performed before, immediately after, and 3 and 12 months after the operation. To quantify the effects of the operation, we collected the following data.

\section{Strabismus angle}

The strabismus angle during fixation with the left eye and during fixation with the right eye was determined by means of the corneal light reflex. Also the cover test and prisms were used before the operation, but exact results were obtained in only $32 \%$ of all cases. After surgery the strabismus angle was always measured with cover test and prisms. In most cases 

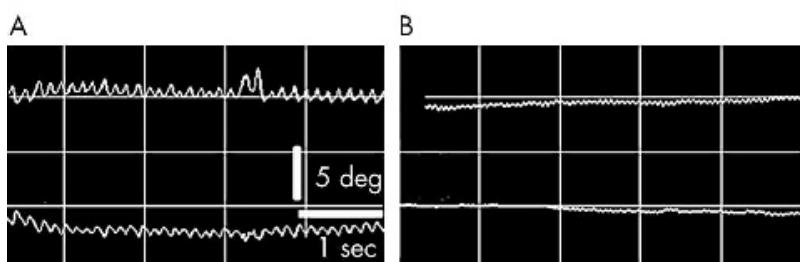

Figure 3 EOG recordings of nystagmus. (A) "Medium" nystagmus before operation. (B) "Very slight" nystagmus after operation. The upper traces show the right eye, the lower traces the left eye. The eyes were examined in primary position.

the strabismus angle for far fixation $(600 \mathrm{~cm})$ was smaller than for near fixation $(40 \mathrm{~cm})$; in a few cases they were about the same. Note, however, that the strabismus angle was variable for each distance.

\section{Nystagmus}

Our patients typically showed latent nystagmus or manifest nystagmus of latens type. One of the main indications for Y-split recession is the presence of nystagmus and of a variable angle strabismus. Owing to the young age of the patients, quantitative recording of the eye movements was difficult. However, by clinical observation it was possible to assess the strength of the nystagmus, as well as the extent of the nystagmus-free field of view. We categorised the nystagmus strength in primary position into one of the following classes: very slight/slight/medium/severe (fig 3).

\section{RESULTS}

\section{Minimum and maximum strabismus angle}

Table 1 shows the average value of the maximum and minimum strabismus angles before, immediately after, and 3 and 12 months after the operation.

Please note that the values in table 1 present the mean values for the whole group. For individual patients, the effect could be much larger: in one subject the Min_Max_Diff was reduced by $27^{\circ}$, and in eight patients over $\overline{20}^{\circ}$. For higher reduction of the variability a higher torque reduction can be used..$^{15}$

Figure 4 presents the distribution of this mean strabismus angle, defined as

\section{Mean strabismus $=($ Max angle + Min angle $) / 2$}

for all 228 subjects, before ("Pre") and 12 months after surgery ("Post"). Please note again that this is the mean strabismus angle, and that the maximum strabismus angle, used for definition of congenital esotropia, is higher (table 1).

\section{Postoperative results}

Based on the results of the surgery and the wellbeing of the patient, we decided in four cases to reoperate on the patient. In one case the effect of the Y-split recession was insufficient,

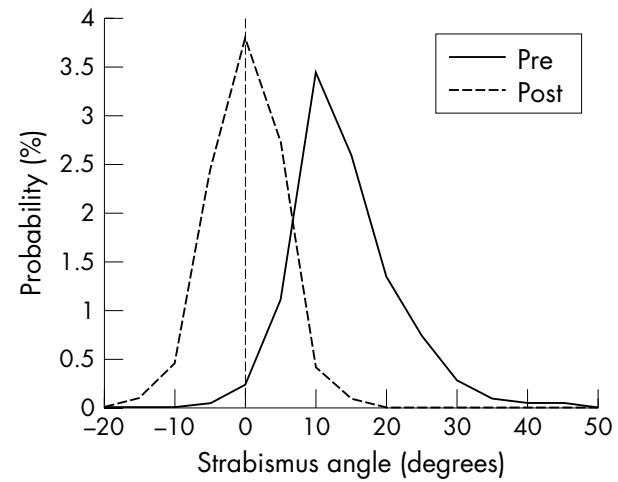

Figure 4 Distribution of the mean strabismus angle before and 12 months after operation.

and the preoperatively alternating head turn in abduction had changed into a constant head turn postoperatively (torticollis). In that patient we decided on an additional Anderson-Kestenbaum operation.

In three patients with a minimal convergence angle above $23^{\circ}$, the effect of the Y-split recession was too low. In those patients we performed in a separate operation an additional resection or plication of one or both lateral rectus muscles. From the fourth case onward this plication has been performed during the same operation. The present study includes 36 cases with one lateral rectus muscle plication, and two cases with both lateral rectus muscles plication. Dosage of plication and resection was determined by the minimal strabismus angle above $23^{\circ}$, in a range between $2.5-$ $7 \mathrm{~mm}$ (4.8 (SD 1.6) $\mathrm{mm}$ ) on one or both lateral rectus muscles. The results are presented in table 2 .

\section{Nystagmus}

In 200 out of 228 patients ( $92 \%$ ), the nystagmus was reduced to slight or very slight after the operation (fig 5). The nystagmus free area started before the operation on average with an adduction of $+8.5^{\circ}$. In other words, the nystagmus affected the primary position and the complete abduction area. After the operation the primary position was nystagmus free in 171 patients $(79 \%)$ : the nystagmus started only at abduction above $-13^{\circ}$. This reduction of the nystagmus field was highly significant.

\section{Other parameters}

In 11 patients $(<5 \%) \quad \mathrm{Y}$-split recession caused a mild adduction deficit. In three patients an overaction of the superior oblique was observed after Y-split recession. Preoperative malfunctions of the oblique muscles, which occurred in 10 patients, were not affected by Y-split recession. Therefore, in three patients an additional recession of one inferior oblique muscle, and in seven patients of both, was necessary.

Table 1 Results strabismus. All values are given in degrees, and the corresponding values present mean (SD)

\begin{tabular}{lllll}
\hline & Before operation & $\begin{array}{l}\text { Immediately after } \\
\text { operation }\end{array}$ & $\begin{array}{l}\text { 3 months after } \\
\text { operation }\end{array}$ & $\begin{array}{l}\mathbf{1 2} \text { months after } \\
\text { operation }\end{array}$ \\
\hline Max angle (degrees) & $18.5(8.5)$ & $5(5)$ & $3.5(5.5)$ & $2(5)$ \\
Min angle (degrees) & $10.5(7)$ & $2(4.5)$ & $1(4.5)$ & $-1(5)$ \\
Min_Max_Diff (degrees) & $8(5.5)$ & $3(3.5)$ & $2.5(3.5)$ & $2.5(3)$ \\
\hline
\end{tabular}

"Min_Max_Diff" of the strabismus angle was defined as the difference between "Max angle" and "Min angle." In addition to the clear reduction of the mean strabismus, also the difference between the maximum and minimum strabismus angle decreases. All these decreases were highly significant $(p<0.01)$. 
Table 2 Comparison of 36 patients with and 192 patients without additional lateral rectus surgery (mean (SD))

\begin{tabular}{lll}
\hline & $\begin{array}{l}\text { Without lateral rectus } \\
\text { surgery }\end{array}$ & With lateral rectus surgery \\
\hline Max angle - preop (degrees) & $18.5(8.5)$ & $34.3(6.9)$ \\
Max angle - 12 months postop (degrees) & $1.6(5.1)$ & $3.9(4.8)$ \\
Min angle - preop (degrees) & $10.5(7)$ & $26.7(7.2)$ \\
Min angle - 12 months postop (degrees) & $-0.8(5.1)$ & $0.7(5.5)$ \\
\hline
\end{tabular}

None of the 228 patients treated with Y-split recession showed any of the complications reported with the Cüppers operation: detachment of the retina, scleral perforation, significant globe retraction, or significant reduction of globe movement.

\section{DISCUSSION}

The majority of patients presenting with strabismus have a constant strabismus angle. In those cases a recession and resection of one or more of the extraocular muscles is usually adequate to treat the problem. In contrast, some subjects present with a large variability in the nystagmus angle, regardless of the fixation distance. This includes patients with "infantile esotropia," also referred to as "congenital esotropia," and patients with "fixed" or "constant congenital" esotropia. The latter group is also referred to as "nystagmus blocking syndrome," as the strabismus angle is fixed in adduction for far and for near fixations. We decided to treat the patients only after they completed amblyopic therapy and exact clinical examination. Patients affected with other ophthalmic diseases like convergence excess and a pathological accommodative situation were excluded from the study. In all these subjects a simple muscle recession or resection is not sufficient: a recession or resection eliminates the minimal strabismus angle by shifting the eye to less esotropia; the variability of the strabismus angle however is not affected. Therefore, this technique is not commonly used in Europe, and instead Cüppers' Fadenoperation is often applied to treat variable strabismus angle. By reducing the torque, the Fadenoperation decreases the effect of the muscle and therefore the variability of the strabismus.

The effect of Y-split recession is similar to the effect of Cüppers. But while the effect of the Fadenoperation depends on the eye position (more incomitance in sideward gaze), the torque reduction is fairly constant for Y-split recessions (see fig 5 of the companion paper ${ }^{1}$ ). Splitting of the rectus muscles has been suggested previously to stabilise globe movement in case of Duanès retraction syndrome. ${ }^{16}{ }^{17}$ In that case one or more muscles are split to enlarge the insertion area on the

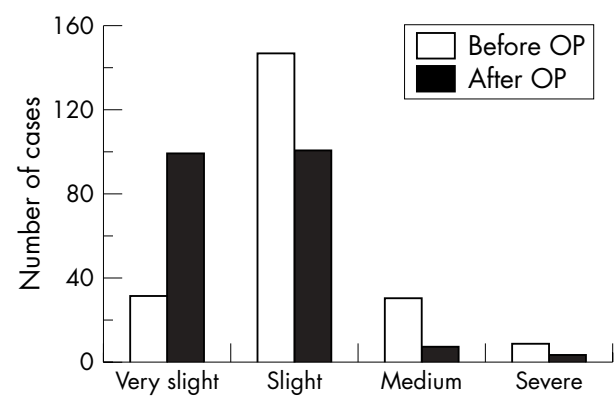

Figure 5 Distribution of the nystagmus: Y-split recession led to a clear reduction of nystagmus in all subjects. The definitions of the different types are given in the text. globe surface. This enlargement of the insertion area can stabilise globe movement in the pulling direction of the muscle, and prevent undesired movements perpendicular to it. The effects of such an operation on the reduction of the muscle torque, which also occurred in those operations, have not been exploited up to now. The Y-split recession operation is specifically aimed at applying that effect. None of our patients showed a significant loss of motility in upgaze and downgaze after that operation. The main effect of the operation was in the change of strabismus angle and nystagmus. The maximum strabismus angle, at near and at far distance, was reduced through the torque reduction. Using a simple computer based model of the oculomotor plant, ${ }^{18}$ the surgical parameters given in figure 1 were chosen such that the torque reduction in the primary position was similar to the parameters typically suggested for the corresponding Cüppers operation. ${ }^{4-7}$ These suggestions were verified by the positive surgical results in our group of 228 patients. The minimum strabismus angle ("static strabismus angle") could be adjusted through the muscle recession that is inherent in the Y-split recession technique. If required, a higher recession of the eye muscle is possible.

In addition to the reduction of the nystagmus, the visual acuity, which had been optimised with occlusion therapy before the operation, improved after the operation, with the same or less occlusion therapy. This postoperational improvement of visual acuity is not only a direct result because of Ysplit recession, but also an accumulation of additional effects: the strabismus angle is decreased, and nystagmus in the primary position reduced or eliminated. None of the 228 patients operated with Y-split recession showed any of the effects that have been reported with the Cüppers operation: movement inhibition and increased incomitance in the direction of operated muscle traction; globe retraction in far eccentric eye positions; or scleral perforation and retinal detachment, caused by the posterior suture fixation (especially in aphakic, highly myopic eyes). The small side effects from the combined eye muscle surgery, like a slight remaining squint, can be treated by additional resection or plication of one or two rectus muscles.

The connective tissues that constrain the muscle to a path close to the orbital wall, and are often referred to as "muscle pulleys," have recently received a lot of attention, and have turned out to be highly important for orthophoria also in a tertiary gaze position. ${ }^{19}$ As shown in figure 1 , the Y-split recession technique does not disturb the pulley structures of the extraocular rectus muscles: for splitting the muscle it is only necessary to "tunnel" the retinaculum ligament. In contrast, the Cüppers operation requires a surgical approach much more posterior. It also fixes the orbital layer structures to the global layers, therefore retracting ("clinging") these structures and leading to an increasingly incomitant effect in adduction. It has been speculated that if a sharp preparation is used, separating the orbital layer from global layer, and only the global layer is sutured to the globe, mechanical restrictions should be minimised, especially in adduction. 
However, the stabilising effect of muscle pulleys would be reduced. ${ }^{20} 21$ In contrast, other publications do not consider pulleys, or believe that the effect of the muscle pulleys are negligible. ${ }^{22} 23$

Theoretically it is possible to modify every rectus muscle with this technique. The effect of Y-split recession on the superior oblique muscle would be quite different from its effect on rectus muscles ${ }^{24}$ : it should be similar to the effect of the Harada-Ito surgery, in which an oblique muscle is split and stretched..$^{25}{ }^{26}$ We have not applied this technique to the inferior oblique, since the inferior oblique is somewhat different from the other extraocular muscles: the inferior oblique and inferior rectus are tightly connected through Lockwood's ligament, and the inferior oblique is very short. And because of the far anterior insertion, the innervation and trophic supply of the inferior oblique could easily be affected.

In summary, the Y-split recession technique provides for a large group of patients an efficient way to reduce the torque of one or more rectus muscles. In our experience, the reduced invasiveness is highly beneficial to the patient, especially in infantile esotropia.

\section{Note}

All data presented above are from a clinical study. The responsibility for surgical results with the Y-split technique lies with the operating physician. Since any change in the surgical technique can affect the results of the operation, we suggest that this surgery should only be performed by a physician with sufficient experience. For detailed questions, please contact the authors. The authors do not assume any responsibility for damage or injury to person or property through application of the Y-split technique.

\section{ACKNOWLEDGEMENTS}

We thank $\mathrm{H}$ Hametner who wrote the computer program that allowed us to find the appropriate surgical parameters.

\section{Authors' affiliations \\ R Hoerantner, S Priglinger, Krankenhaus der Barmherzigen Brüder Linz, Austria \\ T Haslwanter, Department of Neurology, University Hospital Zürich, Switzerland and Upper Austrian Research, Medical Informatics, Linz, Austria}

Correspondence to: Thomas Haslwanter, PhD, Upper Austrian Research $\mathrm{GmbH}$, Medical-Informatics, Softwarepark Hagenberg, Hauptstrasse 99, A-4232 Hagenberg, Austria; thomas.haslwanter@uar.at

Accepted for publication 9 April 2004

\section{REFERENCES}

1 Haslwanter T, Hoerantner R, Priglinger S. Reduction of ocular muscle power by splitting of the rectus muscle I: Biomechanics. Br J Ophthalmol 2004;88:1403-8.

2 Helveston EM. Surgical management of strabismus: an atlas of strabismus surgery. St Louis: Mosby, 1993:422-3.

3 Stager DR, Weakley DR Jr, Everett $M$, et al. Delayed consecutive exotropia following 7-millimeter bilateral medial rectus recession for congenital esotropia. J Pediatr Ophthalmol Strabismus 1994;31:147-50.

4 Cüppers C. Korrektur der Horizontalabweichung. 1972.

5 Cüppers C. Moderne Schielbehandlung. Klin Monatsbl Augenheilkd 1956;129:579-604.

6 Cüppers C. Grenzen und Möglichkeiten der pleoptischen Therapie. Klin Monatsbl Augenheilkd 1961;381:33.

7 Cüppers C. Some reflections on the possibility of influencing the pathological fixation act. Ann Roy Coll Surg Engl 1966;38:308-25.

8 Cüppers C. The so-called Fadenoperation. Second ISA congress, 1974.

9 Stein A, Sradj N. Six years of experience with structural dissociated surgery. Transactions 19th Meeting European Strabismological Association, 1991.

10 Thomas Ch. Erfahrungen mit der Fadenoperation nach Cüppers. 1977.

11 Alio JL, Faci A. Fundus changes following faden operation. Arch Ophthalmol 1984;102:211-13.

12 Bialasiewicz AA, Ruprecht KW, Naumann GO. Staphylococcal endophthalmitis following squint surgery. Klin Monatsbl Augenheilkd 1990; 196:86-8.

13 Bialasiewicz AA, Ruprecht KW, Naumann GO. Staphylococcal endophthalmitis following squint surgery. Klin Monatsbl Augenheilkd 1990;196:86-8.

14 Clark RA, Miller JM, Demer JL. Location and stability of rectus muscle pulleys. Muscle paths as a function of gaze. Invest Ophthalmol Vis Sci 1997; 38:227-40.

15 Roggenkämper P, Sipp V. Bulblänge und Dosierung der Fadenoperation. 1980.

16 Das JC, Chaudhuri Z, Bhomaj S, et al. Lateral rectus split in the management of Duane's retraction syndrome. Ophthalmic Surg Lasers 2000;31:499-501.

17 Von Noorden GK. Recession of both horizontal recti muscles in Duane's retraction syndrome with elevation and depression of the adducted eye. Am J Ophthalmol 1992;114:311-13.

18 Priglinger S, Hametner H. Operative Methoden zur Behandlung des schwankenden Schielwinkels. Mathematische ZusammenhängeComputergestützte Operatioinsplanung. Spektrum Augenheilkd 1994;8:162-75.

19 Demer JL, Miller JM, Poukens V. Surgical implications of the rectus extraocular muscle pulleys. J Pediatr Ophthalmol Strabismus 1996;33:208-18.

20 Clark RA, Isenberg SJ, Rosenbaum AL, et al. Posterior fixation sutures: a revised mechanical explanation for the fadenoperation based on rectus extraocular muscle pulleys. Am J Ophthalmol 1999;128:702-14.

21 Clark RA, Demer JL. Rectus extraocular muscle pulley displacement after surgical transposition and posterior fixation for treatment of paralytic strabismus. Am J Ophthalmol 2002;133:119-28.

22 Mühlendyck H, Linnen HJ. Die operative Behandlung nystagmusbedingter schwankender Schielwinkel mit der Fadenoperation nach Cüppers. Klein Monatsbl Augenheilkd 1975;167:273.

23 De Decker W. In: Lennerstrand G, eds. Functional basis of ocular motility disorders. Oxford: Pergamon Press, 1982:173.

24 Priglinger S, Hametner H, Haslwanter T. Functional topography as a guideline for differential diagnosis of vertical eye movement disorders and oblique muscle surgery. Spektrum Augenheilkd 2001;15:16-26.

25 Harada M, Ito Y. Surgical correction of cyclotropia. Jpn J Ophthalmol 1964;8:88

26 Fell P. Management of paralytic strabismus. Br J Ophthalmol 1974;58:255. 